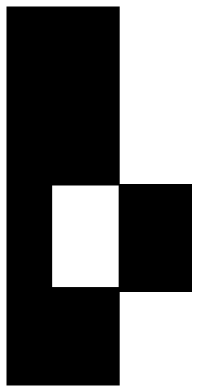

\title{
Writing in Conservation Biology: Searching for an Interdisciplinary Rhetoric? ${ }^{1}$
}

\author{
Betty Samraj \\ San Diego State University \\ John M. Swales \\ The University of Michigan
}

Writing in a new or emergent field, especially one with an interdisciplinary ethos, is generally reckoned to be problematic; certainly more so than in areas where there has been a historic accrual of conventions for writers and expectations for readers. Bazerman (1988) argues, for example, that political science "has yet to forge a consistent nature" (p. 288) because of its struggle to accommodate science and political philosophy. Similarly, Wignell (1998) ascribes the heterogeneous nature of sociological discourse to a lack of synthesis between the discourses of science and the humanities. More self-evidently interdisciplinary fields tend to be newer and smaller. Commenting on a particularly complex web of intertextual links in urban planning, Dunlap (1992) notes that:

the newness of the field, its changing mandate, its eclectic disciplinary identity, its needs to communicate with both academics and those immersed in practice, its possibly precarious future - all affect planners' conceptions of themselves and their discourse. (p. 215)

On an individual level, Journet (1990, 1993) offers telling historical case studies of pioneering struggles for interdisciplinary discourse. In the earlier paper, she states that to achieve an emergent discourse of neuropsychiatry Luria and Sacks "blurred the genres of neurological and psychological writing, combining the analytic exposition of neurological data with psychological narrative and story" (p. 182). In the later one, Journet analyzes the role of Jelliffe (1866-1945) in creating a new genre of the neurological case study by fusing exposition and narrative and by combining abstract generalizations with the unique specifics of particular patients.

The growing importance of work in interdisciplinary rhetoric is clearly seen by a fairly recent issue of Social Epistemology (1995) devoted to this topic. The four case studies reported therein focus on texts straddling 
genetics and natural history, genetics and paleontology, paleontology, statistics, and astrophysics, and on an unsuccessful interdisciplinary text in geophysics. These studies explain the interdisciplinary nature of these texts not just in terms of rhetorical constructs such as the ethos created or the types of appeals made to a heterogeneous audience, but also in terms of more specific textual features such as the presence of definitions and explanations, and the types of intertextual links made by the authors (Ceccarelli, 1995; Journet, 1995; Sullivan, 1995; FalerSweany 1995).

Environmental discourse, the focus of the present study, has also been examined in terms of its complex and conflicting rhetorical exigencies, perhaps in response to the fact that such discourse on the environment somehow seems central to late twentieth century political, scientific and educational life. The genre of environmental impact statements has come under particular scrutiny (Miller, 1980, 1984; Killingsworth and Palmer, 1992a, 1992b; Killingsworth and Steffens, 1989). Miller(1984, p. 164) claimed that "the imperfect fusion of scientific, legal and administrative elements" prevents an interpretation of the documents as meaningful rhetorical action. Killingsworth and Palmer's Ecospeak (1992a), the only monograph known to us on environmental discourse, traces the history of environmental rhetoric and provides a comprehensive description of various types of environmental writing ranging from newspaper reports to research articles. The results of textual analyses of several different genres are related to the ideological beliefs of various environmental constituencies such as "deep" ecologists, economists, scientific ecologists, and government officials. Generic comparison is carried out in terms of such oppositions as natural history versus theoretical science, familiar language versus scientific language, human interest versus natural science, applied research versus basic research, and "gray" literature versus refereed literature. Killingsworth and Palmer thus provide an interesting picture of "several distinct ethical and epistemological perspectives on environmental issues" (1992a, p. 11) via the analysis of texts produced by groups involved in environmental affairs.

More recently there has been an edited volume on environmental rhetoric (Herndl and Brown, 1996), which mainly includes studies of various types of environmental oral and written discourses such as apocalyptic narratives, nature writing, risk communication, and public discussion sections in hearings. These studies provide insights into the rhetorical strategies employed in each type of environmental discourse and the functions of these strategies in the contexts in which the texts are used. Although, like Ecospeak, most of the articles are not directly concerned with the possible interdisciplinarity of the texts studied, two of the studies do point to the hybrid nature of one common type of environmental writing, nature writing. Slovic (1996) presents an account of this genre's 
combination of natural history and spiritual autobiography and considers the tensions that arise from the use of rhapsodic and jeremiadic rhetorical modes within the same text. To a smaller degree, the interdisciplinary nature of Leopold's nature writing is also discussed by Ulman (1996), who focuses on the complex personae, and ethos created by Leopold in the experience-based ecological essays he wrote.

As we have seen, several different types of environmental discourse have been discussed with clarity and lucidity in the literature. Even so, researchers in this area have had so far little to say about the discourses produced by students in environmental science. In principle, therefore, investigations of student writing in environmental fields would add to the little that is known about the writing practices students engage in when seeking to enter an interdisciplinary community, especially if they could be directly compared with the writings of post-apprentices in the same area. To date, only a few studies on writing in interdisciplinary courses at the graduate level have apparently been conducted. One is Henry's (1994) analysis of writing in a class on landscape design theory, triangulated to encompass the students' written texts, the comments of the instructor and the graduate teaching assistant, and the uncertain history of the field. Another is that of Dunlap (1992), referred to earlier, which discusses the problems that students in urban planning face as a result of the contradictory and yet-to-be established norms.

We have selected five papers to illustrate the rhetorical exigencies of interdisciplinary writing in the environmental area. All five come from the field of Conservation Biology. There are three final term papers written by students taking a 500-level masters course in this subject (out of a class enrollment of 25). The first of the student texts presented was judged by the instructor as being unsuccessful, the other two as being broadly successful. The fourth text is a book chapter by the instructor herself, at the time of writing a relatively junior professor (Root, 1993). The final text, which is partly based on the fourth one, is co-authored by the instructor and a well-known climatologist from another institution (Root \& Schneider, 1993).

Thus, we offer a "ladder" of five texts onto which we can map textual and contextual analyses. In so doing, we can explore how attempts at interdisciplinary "bridging" are variously made, such as via the integrating of theory and practice, of scientific finding and environmental recommendation, of small-scale and large-scale studies, or of concepts drawn from two or more fields of inquiry. However, before discussing the texts themselves, we feel it may be helpful to situate them within the evolving perceptions of graduate education in our research site, a School of Natural Resources and the Environment (henceforth SNRE) at a large public midwestern university, and how these perceptions relate (or do not relate) 
to the struggles toward interdisciplinary status in the sub-field of conservation biology.

\section{The Emergence of Environmental Studies}

Environmental studies or environmental science has a somewhat confusing intellectual history, brought about in part by differing views as to whether ecology is best conceived as a theoretical or applied field. However, one common conceptualization is to see environmental science as having developed from ecology, itself an offspring of the two original branches of natural history, botany and zoology. As the field of ecology has developed, it has turned its attention to more applied concerns, at least partly in response to mounting evidence of environmental change and degradation in many areas of the world. So today, environmental science, from an original base in ecology/applied ecology, now encompasses fields such as resource economics, resource policy, environmental ethics, and conservation biology.

However, this potted intellectual history (or any variant of it) is not in any close or isometric relation with the history of environmental education at our research site. While there have doubtless been intellectual influences, a stronger set of determinants of change appears to have been shifts in the occupational, administrative and legislative landscape. These latter effects are well captured in the changes in nomenclature for this activity during this century:

1901 - Department of Forestry formed.

1927 - School of Forestry and Conservation established.

1950 - A separate Department of Conservation created and the school renamed the School of Natural Resources.

1966 - Department of Landscape Architecture transferred from the School of Architecture and Design.

1970 - Departments disbanded and replaced by Concentrations in Resource Ecology and Management, Resource Policy and Behavior, Forestry, and Landscape Architecture.

1992 - Name of school changed to the School of Natural Resources and the Environment (SNRE).

These changes in organizational structure and self-designation seem to show the school adapting primarily to changing job opportunities for its masters and doctoral students and only secondarily to increasingly inter-connected ways of presenting and producing professional environmental knowledge. This evolution thus privileges exogenous interdisciplinarity, which originates outside the university because of problems in society, rather than endogenous interdisciplinarity, which is con- 
cerned with the production of new knowledge per se (Klein, 1990). However, it is endogenous interdisciplinarity which was highlighted by the dean of SNRE in the 1991/92 Annual Report, which could claim, with that level of modesty characteristic of the genre, that "For more than 50 years the School has set the standard in teaching and research, and we're a model of interdisciplinary learning and teaching, with biologists, foresters, social scientists and landscape architects all under one roof' (p. 42). But having diverse types housed "all under one roof" does not, of itself, interdisciplinarity make. While many in SNRE-type institutions have recognized that environmental studies has needed to develop into an interdisciplinary field, if only because environmental problems are "multi-faceted" (Hay, Todd \& Russell, 1986) and do not come in "disciplinary-shaped blocks" (Petrie, 1992, p. 305), the ways in which this has worked out in educational practice seem for the most part indirect. As one of our instructor informants commented: "The hope is that students acquire knowledge for different disciplines while they are here, and they get some skills that let them function in a workplace that forces them to do some integration" (interview data). Interdisciplinarity thus seems more of an occupational opportunity than of a directly-structured educational goal.

So, in SNRE itself there is ostensibly considerable waving of the interdisciplinary flag, such as the use in many courses of guest lecturers from other disciplines. However, in our experience, these occasional migrations rarely impact much on assigned readings, on the ethos of the main lecture series, or on the types of course assignments expected of the students. For the most part, the masters students seem most akin to searovers voyaging from one disciplinary course-island to another, accumulating knowledge capital of a different currency on each.

That said, at least one of the so-called interdisciplinary courses in this institution announced itself as taking its "interdisciplinary responsibilities" seriously: the 517 class in Conservation Biology taught by Terry, a recently-promoted associate professor. It is perhaps not surprising that a stronger integrative ethos should have been located in this course (rather than in Resource Policy or Wildlife Behavior), since conservation biology is a "crisis discipline" whose goal is to "provide principles and tools for preserving biological diversity" (Soule, 1985, p. 727) and acts as "science in the service of conservation" (Noss, 1993, p. 215).

Conservation biology thus appears, at least potentially, to be triply articulated: it attempts to co-manage theory and practice; it is catholic in its incorporation of elements ranging from pure science to social science; and it is integrative. In terms of this last, it is worth noting Petrie's (1992) distinction between multidisciplinary and interdisciplinary research, where the former is additive rather than integrative and the latter involves a change in some key elements of disciplinarian's use of concepts and tools 
and hence is integrative. This putatively seems to be the position adopted by Terry, the central figure in our story; for her, multidisciplinary activities in SNRE and elsewhere represent "the bare minimum" (Root, 1993, p. 291) and are but a waystage to somewhere else - and somewhere better. This paper, therefore, focuses on the 517 class in Conservation Biology and selected writings of the instructor.

\section{Observations on the Primary Research Site}

Terry stated at the beginning of the semester that the goal of her 517 course was to move the students from a multidisciplinary perspective to an interdisciplinary one. Though she did not explicitly define the difference between these two stances, she did mention early in the semester the different disciplines that contribute to conservation biology, such as botany, ecology, economics, and politics, and also pointed out that interdisciplinary study is difficult to undertake as integration is never taught (c.f. Petrie, 1992). Even though we could recognize that Terry perceived interdisciplinarity to be integrative and saw this to be the goal of the course, the course itself in reality bore a greater resemblance to the encyclopedic approach associated with multidisciplinary education, as characterized by the additive juxtaposition of disciplines (Klein, 1990, p. 56). This course indeed included topics ranging from biological issues such as calculating allele frequencies to social ones such as overpopulation. There were also several guest lectures by lecturers from such fields as cultural anthropology and paleontology. However, no interdisciplinary synthesis was provided either throughout the course or at the very end.

There was only one major writing assignment for this course. A list of possible topics was given to the students and they were instructed that papers "could not be all policy and had to be at least 50\% biology." Terry also discussed the overall organization of their papers, making it clear that they needed to specify a problem in the introduction and that the writing had to be concise. Though Terry spent class time discussing the nature of the students' peer critiques, the structure of their papers, and features of the oral presentation of their papers, none of her comments addressed the interdisciplinary dimension of the research papers.

The instructor allowed the first author to attend all her lectures for a semester, make copies of evaluated student papers (with the students' consent), talk to the students about the papers they produced for the course, and discuss the papers and grading criteria with her teaching assistant and her. There were two principal reasons for attending all the classes. First, it allowed the first researcher to become familiar with the subject matter so that there would be less content ignorance when analyzing the written texts. Second, attending the classes provided important information on the ways in which the writing task was framed and dis- 
cussed, allowing the investigator to explore possible connections between " classroom contexts, students' texts and professor's responses" (Prior, 1992, p. 282).

22 out of the 25 students taking this course agreed to be part of the study. (See Samraj 1995 for a complete discussion of the methodology and full analysis of all 22 texts.) Copies of the graded papers were made before they were returned to the students. Thus, we had access to the instructor's and teaching assistant's assessment of the papers, including the grade or score, as well as to the written comments and evaluations which proved indispensable in providing the specialist's view on the strengths and weaknesses of each text. In fact, none of our own judgments was used to determine the success of the papers written in this course.

\section{Five Texts}

The first three texts are term papers from the course in Conservation Biology, the first judged to be unsuccessful (written by Jessie), and the other two deemed successful though in slightly different ways (written by Adele and Ned). The last two texts consist of a book chapter by the instructor (Root, 1993) and an article co-authored by the instructor and a climatologist (Root and Schneider, 1993). None of the five texts has the format of standard research papers, such as an Introduction-MethodResults-Discussion (IMRD) section arrangement (Hill et al., 1982). Although all five certainly contain passages that reflect scientific style as discussed by Bazerman (1988), Swales (1990) and Montgomery (1996), they also contain elements of exhortation and advocacy. Because the student Conservation Biology papers were not reporting original research, we might not expect them to be organized like published research articles, but to have more of the character of reviews. Indeed, Johns (1995) has usefully shown that coursework papers, which she refers to as classroom genres, have different communicative purposes compared to published research articles and this results in different overall organizations. Likewise, the published papers in our study also do not have the IMRD structure since they also do not report new empirical findings, but rather present their own previous work to readjust research priorities.

\section{Jessie}

Jessie's paper illustrates some of the difficulties graduate students without field experience have with producing interdisciplinary writing. Her paper "Influence of Sex Ratios on Fitness in Reptiles" mainly discusses the explanations that have been offered by researchers for the persistence of skewed sex ratios in adult reptile populations. This paper refers exclusively to articles in biology and ecology, there apparently being no references to sources from the social sciences. It looks at first sight 
as though this is a disciplinary text and not an interdisciplinary one; in fact, Jessie's paper, in both topic and treatment, seems to have migrated from one of the other graduate courses in SNRE such as Wildlife Behavior and Ecology.

Jessie's paper can be said to have the Problem-Solution structure, by far the most popular type of organization among the student papers (18 out of 22) from Conservation Biology. However, the "Problem" in her paper does not concern some factor surrounding a species' diminution or problems with solutions currently being offered for a conservation problem. Rather, in her paper she problematizes the theoretical explanations that have been offered for skewed sex ratios in reptiles (as indicated by her heavy use of negative evaluation). Though skewed sex ratios in a species is undoubtedly a conservation concern, her paper is organized around the problem of how ecological theories might or might not account for such behavior instead of the nature and extent to which this phenomenon is a problem to conservation. Consider the opening sentences of her paper:

Many taxa are known to exhibit significantly skewed sex ratios in adult populations (Mrosovsky and Provancha 1991). This clearly contradicts Fisherian theory, which suggests that an equilibrium sex ratio of one male to one female will be maintained by selection (Fisher 1930). (Jessie 1-2)

The problem presented in the first sentence is thus immediately related to a theoretical consideration. This rhetorical strategy continues throughout the paper, resulting in an organization centered around three explanations that have been offered for skewed sex ratios in reptiles. The structure of this paper thus appears similar to that of review papers with a cyclical structure (Belcher, 1995, Samraj, 1995), where an aspect of a theory or a finding of some study is reported and then commented on. This pattern of description-comment can be seen in the following two short extracts (our emphases):

Several researchers have observed that some species with TSD lay clutches of eggs which prove to be nearly or completely unisexual (Mrosovsky and Provancha 1991; Vogt 1984). This is advantageous if both the cost and potential of brother-sister matings are high....Ewert and Nelson have suggested that some freshwater reptiles (such as turtles or crocodilians) might be regularly restricted to this breeding situation. For instance, a population of turtles may consist only of those found in a single pond or a small group of ponds, and in fact most such populations of turtles are female 
biased (Schwarzkopf and Brooks 1987). In such cases, inbreeding would be common, and a loss of herterozygosity should be expected in wild populations. (Jessie 13-14, 25-27)

As might be expected, the Problem-Solution Conservation Biology papers written by students generally end with recommendations for conservation actions as well as recommendations for future research. The extract below contains the last two paragraphs of Jessie's paper, where her recommendations appear (our emphases):

If this happens, then conservation efforts will need to focus on maintaining historically correct sex ratios of adults. Sex ratios of juveniles can be easily manipulated by controlling the incubation temperature in a hatchery. Size of hatchlings may be controlled as well by other factors, such as moisture, which will not influence sex determination (Ast, unpublished). Thus, unbalanced sex ratios due to differential fitness could be overcome by careful control of hatchery conditions.

Unfortunately, it is not known whether the juvenile sex ratio will remain stable up to and through maturity. Furthermore, true sex ratios of wild adult populations are largely unknown, and whether present-day sex ratios are historically correct is also unknown. Any attempt to change sex ratios, without a knowledge of the forces responsible for them in the first place, could have severe consequences for the population. Further investigation into the evolutionary and environmental factors producing skewed sex ratios is clearly needed. (Jessie 54-61)

In the first paragraph conservation effort is advocated. The first sentence states in general the need to maintain historically correct ratios. The second sentence then tells the reader how this can be done, that is, by controlling the incubation temperature. The final sentence restates in general terms what can be done to overcome unbalanced sex ratios. Unfortunately for Jessie, the author claim in the final paragraph in this paper makes it clear that the conservation effort advocated is in fact based on assumptions that are unverified. In consequence the writer then retreats to stating the need for further research, since she has in fact already negatively evaluated the action advocated - to change sex ratios - in the previous paragraph. This paper, then, does not in fact suggest any conservation action, let alone come up with a conservation scheme (because the only conservation action advocated is negatively evaluated). 
This lack of resolution doubtless underlies Terry's final written comment that "the link to conservation was ... not too strong."

Even with these brief extracts, we hope to have communicated that Jessie establishes her persona in the text mainly through her discussion of implications of ecological theories and not by recommendations for conservation actions. Though the paper appears to have the Problem-Solution structure that characterizes many of the more successful student Conservation Biology papers, a closer analysis of the text reveals that the problematization concerns theoretical matters and not issues surrounding a species' diminution. In this paper, Jesse has yet to cross the bridge from biological science to conservation biology.

Adele

Adele majored in psychology and geography as an undergraduate and, in contrast to Jessie, produced a paper that was quite highly ranked in this course. Her paper focuses on the importance of educating and involving the public in wildlife migration. In doing so, she does not only discuss biological factors but also investigates socio-political factors that affect the migration of species, especially of the gray wolf.

Adele's paper is comparable to Jessie's paper in that it too has the Problem-Solution structure. The problem focus of the 18 papers with such an overall organization is typically some factor surrounding a species' diminution or inadequacies with the solutions currently offered for a conservation problem. Most of the papers then go on to deal with solutions to problems faced by an endangered species or concern themselves with debates about possible solutions or lines of action. In consonance with this model, Adele's section headings indicate clearly enough the paper's basic organization in terms of its Problem-Solution structure:

1. Introduction

2. Migration of the Gray Wolf: A Case Study History

Obstacles affecting migration

Overcoming the obstacles affecting migration

3. Global Warming and Wildlife Migration

4. Educating and Involving the Public

5. Conclusion

Further, the Problem-Solution pattern is fleshed out by the fact that section 2 is a case study that itself functions as a problem-solution structure embedded within the larger one.

As already noted, the Problem-Solution Conservation Biology papers written by students generally end with recommendations for conservation actions as well as recommendations for future research. However, 
as can be deduced from the instructor's comments elsewhere in other papers, the recommendations for conservation actions are of primary importance. In Jessie's paper, the failure to establish the basis for making a conservation recommendation is criticized. In other cases in the database, the instructor shows impatience with any suggestion for further research. In one case in a paper on macaws, the instructor reacts to a research recommendation by stating, "If we wait, they all may be extinct," echoing the view that conservation biology is a crisis discipline, where action has to be taken immediately.

Adele does provide a number of recommendations for management actions and thus establishes her stance as a conservationist (in contrast to Jessie):

If global warming occurs rapidly, as predicted, mass migrations of wildlife species may occur, affecting all people, either directly or indirectly. Therefore, it is imperative that wildlife managers become adept at understanding the interactions between humans and migrating wildlife, and at managing not only wildlife recovery programs, but also public education and involvement programs. (Adele 101-103)

As the above extract shows, Adele's paper is suitably integrative. She draws on ecological information concerning the behavior of wolves, information on global warming, and sociological theory on the nature of human behavior in order to present suggestions for the problem of wildlife migration. Some of this is an integration already well-addressed in the conservation biology literature (and in Terry's lectures) but relating sociological theory of human behavior to wildlife behavior is a new synthesis produced by Adele in her attempt to suggest solutions to the conservation problem. It is not surprising that Adele's balanced and competent literature review caused Terry to comment that she had chosen "a good topic" for her paper.

Ned

The third selected paper was written by Ned, a native speaker of Swedish. It stands out from the majority of the student Conservation Biology papers by being one of two case studies produced. It mainly focuses on a Swedish eagle conservation project, but also briefly examines the strengths and weaknesses of other eagle conservation projects in Finland, Scotland and the US before providing recommendations. As both the topic and paper suggest, he has had prior experience in conservation biology. This is a paper that the instructor said should be published, but as far as we have been able to ascertain the student finished his masters program without reworking it for possible publication. 
A main difference between this paper and the Problem-Solution papers lies in its focus on evaluating the conservation strategies used in a specific case study. Both positive and negative local evaluations are made, and these then form the basis for the author's own local management and research recommendations, creating a Situation-Evaluation-Recommendation sequence. Ned's evaluation of actual conservation programs seems to exhibit considerable familiarity with conservation actions taken in the real world in his area of interest. For example, in the excerpt below, Ned reports a positive evaluation of the work of the Swedish Association for the Conservation of Nature (SNF), this evaluation then leading to a closing recommendation:

SNF effectively used media and mobilized volunteers to work on the project. Being the largest environmental organization in Sweden, SNF reaches a large part of the population through its campaigns and publications ... Thus, involvement of NGOs in the planning and implementation of similar projects is likely to be beneficial. (Ned 115-120)

Ned not only presents evaluations and recommendations about actual conservation programs but also is able to add to the disciplinary conversation on biological issues. In one place, Ned makes the claim that a certain piece of biological information, effective population size $(\mathrm{Ne})$ for a species, is important for species management. This claim is supported by the student's own calculations, which are positively evaluated by the professor with an "excellent" and "nice":

Although the major objective of the Project Sea Eagle is to increase the total number of individuals (Helander, 1986d), estimates of $\mathrm{Ne}$ can help in determining critical management decisions. Yet, I have not seen estimates of $\mathrm{Ne}$ in the literature. My own estimates based on data from Helander (1985c:424; 1991b:8), indicate an effective population size of 126 individuals from the Baltic sea population in 1990 (see appendix 1). This number is higher than the $\mathrm{Ne}$ of at least 50 individuals proposed by Frankel and Soule (1981) as the minimum for the short-term preservation of reproductive fitness. However, if calculations of $\mathrm{Ne}$ are made using population numbers from 1990 and juvenile survival rates similar to those before the winter feeding program started, the effective population size is only 36.5 individuals. The supplemental winter feeding may thus create an illusion of unrealistically high $\mathrm{Ne}$ if its effect on juvenile survival is not controlled for in the calculations. In 
the future when deciding on whether there is need to continue the project, calculations of Ne are essential. (Ned 90-96) (our emphasis)

Ned's claims in this paper show him contributing both to the theory and practice of conservation biology. The projected image of a young but experienced practitioner as created by his evaluations of conservation programs is complemented by the image of a biologist able to challenge previous calculations of effective population size and propose more accurate figures of his own. Thus, unlike Adele, Ned does not just provide a number of recommendations based on findings from various disparate fields relevant to the conservation project, but can show a personallyengaged and integrative expertise.

Finally, Ned is particularly successful in intertwining recommendations for research with recommendations for conservation actions. The extract below provides a splendid example of the advocacy of both research and conservation actions (our emphases):

The Baltic Sea and Lappland populations are regarded as separate based on observations of banded birds (Helander, 1990a). Researchers could use electrophoretic studies to further test this assumption and to determine the extent of gene flow. If the low number of birds the last hundred of years has resulted in higher inbreeding than normal, the Swedish sea eagles may face risk of inbreeding depressions. In this case, active translocation of birds or eggs may be necessary. (Ned 84-87)

\section{Terry}

The fourth paper appears as a 13-page chapter in a 1993 volume entitled Biotic Interactions and Global Change, the outcome of an interdisciplinary workshop held in 1991 on San Juan Island (Washington). Terry's paper (Root, 1993) reviews contemporary or near-contemporary studies of climate change, stresses the problematics of rapid temperature increase, inserts within that some empirical data on the likely effects on selected trees and birds, discusses possible ways forward in terms of both species studies and realignments within the scientific community, and closes by reminding that community and those outside it of their joint responsibilities. In some sense, the paper accords well with what the editors say about the volume as a whole: "The book is divided into six sections and includes synthetic literature reviews, critical commentaries, arguments for particular research directions, and the results of original research aimed at the challenge we posed to the authors" (p. 2). However, 
it will become clear that her paper does not do just one of these four things, but rather attempts most of them.

The overall organization of Terry's paper does not lend itself to an easy description. A quick review of the text shows that it is not a research paper of any standard or traditional sense. For instance, it does not display any new empirical data collected and analyzed by the author, and in both choice of section headings and in substance it does not, like the student papers, follow the standard IMRD format. We can see the overall shape of the paper from its section headings (and can note in passing the similarity with Adele's headings):

1. Untitled Introduction (Problem Specification)

2. Ecosystem Response to Rapid Change is Problematic Vegetation

Birds

3. Forecasting Potential Change in Species Communities

4. Outreach

5. Summary

This paper is also similar to Adele's paper in having a Problem-Solution structure with an embedded case study, but here the ornithological findings are taken from a major study by the author herself, rather than gleaned from the literature.

Though Terry instructed her graduate students not to be overly concerned with research recommendations per se, they play a prominent part in her text. However, of the various research recommendations, a substantial proportion deal with the organization of the research effort. Here is one of several examples:

Additionally, the reward system within the scientific community is such that interdisciplinary work is not valued as highly as disciplinary work (e.g. ...). Such barriers are common at the cutting edge of problem-solving, but they must be breached before the complex problems caused by global climate change can be adequately addressed. (Root, 1993, p. 291)

In this way, she concentrates her strongest recommendations for what her own scientific community should be doing and avoids lambasting obvious anti-environmental forces in the wider world. The opportunity she takes to re-frame the discussion within the context of the sociology of environmental science (its reward systems, hierarchical structures and border disputes, etc.) is one presumably that junior graduate students are unlikely to be able to contemplate. Although policy recommendations are not missing from the paper, their secondary status is indicated 
both by their placement (following the research recommendations) in the section "Outreach" and by their lack of specificity.

Terry's article seems to be a mix of an empirical and review paper. It is similar to the papers by Adele and Ned in being grounded in a conservation problem. Though the paper may at first appear to bridge a connection between two fields, climatology and ecology, it mainly deals with the latter. In this chapter, which Terry acknowledges to be "something of a new departure" for her (personal communication), the transitions between research and policy as well as those between macro- and micro-environmental features (such as the climate and the ecology of birds) seem at times incomplete or abrupt (see, in particular, Fig. 1 below). For a resolution of these problems, we turn to the final paper.

\section{Terry and Steve}

Terry's "new departure" paper concentrated on ecological research (showcasing therein her own major ornithological study), prefaced by an opening discussion of global change prognostications and followed by a statement of the implications for environmental research. With the addition of Steve, their two research agendas are brought into conjunction and a paper with a greater degree of interdisciplinarity is produced (Root and Schneider, 1993).

On one level, both published papers can be seen as having the same three linked parts: the environmental problem of global warming with its effects on plants and animals; the problems of mismatch in scale between climatic modeling studies and ecological studies; and the role of the scientific community in solving environmental problems. However, in the joint paper, the object under study (Bazerman, 1988) more obviously transmogrifies from intrinsically environmental concerns to those of how to conduct suitable studies that would enhance the quality of policy making. This agenda first achieves prominence as early as the final paragraph of the Introduction:

The purpose of this paper is to discuss approaches that attempt to reduce the mismatch. We also provide examples of successful "bridging" studies that examine and suggest avenues forfuture work. These studies provide examples of how such work can be used as a foundation upon which the small-scale biological studies and large-scale climatic studies can be coupled to clarify further the possible biological consequences of global climatic change. (Root and Schneider, 1993, p. 258)

A preliminary understanding of the development of this paper from the single-authored one can be obtained from a comparison of the section 
titles of the two papers (see Fig. 1). Two features of the overall organization of the joint paper are evident from the section headings. First, the addition of Steve as co-author has enabled the paper to achieve a carefully-stepped general-specific-general movement characteristic of many research articles (Hill et al., 1982), which we have attempted to display visually in Figure 1. Second, many of the subsections explicitly appear to link climatology and ecology. Note, for example, "Global Warming Scenarios Relevant to Ecosystem Studies" and "Forecasting Large-Scale Vegetational Response to Rapid Climatic Change.”

\section{Figure 1: Announced Structures of Terry and Terry \& Steve}

Terry

Introduction (unlabelled)

Ecosystem response to rapid change is problematic

Vegetation

Birds

Forecasting potential changes in species communities

Outreach

Summary

Terry and Steve

Introduction

Global Warming Scenarios Relevant to Ecosystem Studies

Narrowing the Mismatch between Ecological Studies and

Climatic Model Scales

Unpredictability of Time-Evolving, Transient Climates in

Regional Areas

Forecasting Large-Scale Vegetational Responses to

Rapid Climatic Change

The Importance of Triangle Linkages between Plants, Animals, and Climate on a Large Scale

Specific Example Using Wintering North American Birds

Possible Physiological Constraints Facilitating the

Bird-Climate Linkage

Forecasting Potential Changes in Species Communities:

Integrating Field and Large-Scale Studies

Outreach

Implications for Policy 
A close analysis reveals that Terry and Steve's paper is itself a comprehensive example of a successful "bridging" study that can link micro and macro issues. Most of the first seven sections after the introduction integrate climatology and ecology. The level of integration seen here therefore goes beyond that found in the student papers or the singleauthored paper. Notice the degree of integration in the sentence from the section "The Importance of Triangle Linkages between Plants, Animals, and Climate of a Large Scale"(6):

If the climate changes more rapidly than the dispersal rate of the plants, resulting in extensive die-offs in the South before individuals can disperse and become established in the North, then the ranges of animals relying on these plants could become compressed. (Root and Schneider, 1993, p. 118)

As already mentioned, a significant proportion of the recommendations in this paper pertain to research. Not only are recommendations made concerning individual research areas, but a re-visioning of research activities as a whole is also advocated. Advocacy of this research to reduce "mismatch" and to construct "bridges" is interestingly similar to Bazerman's conclusions about the writer of the sociology paper in Shaping Written Knowledge (1988), namely, the sociologist's need to persuade the readers of not just "the specific claims of the essay, but of the author's larger framework of thought in which his claims are placed" (1988, p. 35). Bazerman goes on to note that in such a situation language "must be carefully shaped by the author to turn his own vision into a shared one of the discipline" and also seems to imply that this trait may be more common in the social sciences where authors are noted for "a point of view or method of perception rather than a specific claim" (ibid, p. 39). Likewise, an attempt to project a visionary search for a discipline-combining methodology would seem to be part of author persona in conservation biology, at least at the professorial level.

\section{Discussion}

If both student and published papers are intended to be interdisciplinary, we can then return to the most important of our research questions, "Is a different sort of interdisciplinarity seen in these two sets of texts?" Interdisciplinarity does indeed seem to be conceived of rather differently. The successful student papers achieve interdisciplinarity primarily in exogenous terms, that is, in terms of problems in the real world. Interdisciplinarity here is almost synonymous to the application of theory to practice. The published papers, on the other hand, move from exog- 
enous interdisciplinarity to endogenous interdisciplinarity, which is concerned with the production of new knowledge, in this case, an integration of ecology and climatology. Interdisciplinarity in the published papers also concerns integrating the goals and activities of scientists and policy makers. Finally, Terry and Steve's paper stands apart from the other papers through its explicit discussion of interdisciplinarity in environmental science.

Only the paper by Terry and Steve does seem, in its creative remaking of itself out of two separate single-authored chapters, to be constitutive of interdisciplinarity when described as an "overarching conceptualization that transcends the epistemological and methodological perspectives of the constituent disciplines" (FalerSweany, 1995, p. 169). And it will doubtless not have escaped the reader's notice that this high-achieving paper was the only one to be co-authored. Therefore, if this study has a modest practical message for Terry and her teaching of the 517 class it would be for her to reflect upon the possibility of encouraging collaborative writing, especially in cases where students coming from differing disciplinary backgrounds can be paired up.

Terry's Conservation Biology course took its interdisciplinary ambitions seriously, even though interdisciplinarity as measured by current concepts such as "interpenetration" (Fuller, 1993) was not a distinctive or pervasive feature of the actual student writing. This seriousness on Terry's part is very apparent in the following E-mail response to our query about her educational practice:

I agree it is a learned skill to be able to integrate disparate info in a way that addresses a real world problem. Most undergrads are never taught how to do this. Indeed they are taught to narrowly examine problems and hence have not learnt to "see" connections. In my class and with the assignments, I am trying to teach them to broaden, make linkages between fields and info in the various fields. The first step is to see how "basic" research (like population genetics), that is quite disciplinary-focused, can be broadened to address real world problems. That is hard and takes new training (not only of students, but of those disciplinary folks that are threatened by the broadening (they call it "sloppy science") of their work).

It indeed turned out hard for some of her SNRE 517 class.

As we have seen, in the case of our two professional scientists, one way to attain interdisciplinarity lies in being able to form a bridge (Journet, 1995, p. 123) between the "micro" and the "macro" by creating a new kind 
of scalar continuum onto which research of very different types can be coherently attached (as shown in Fig. 1). However, this was a strictly professorial achievement, and it turns out not surprising that the graduate students were unable to recognize, problematize or scaffold such mismatches of scale in their own papers. Although the issue of scale mismatches among studies conducted in various subfields of environmental science was discussed in one class very early in the semester, the methodological and epistemological problems created by disjunct scales were not raised when instructors from such fields as anthropology, sociology, paleontology, and policy science presented guest lectures in the class. Nor was class time spent on relating the epistemologies of the contributing disciplines after the talks had been given. The graduate students then didn't have much of an act to follow. We have identified scalar continuity as one solution to the interdisciplinarity problem. In fact, Terry and Steve promote their scalar bridges as methodological necessities for solving important real world problems, rather than offering them as mere connective devices supporting the framework of some kind of integrative review paper. Thus, theory and practice are also aligned. The students revealed through their course papers that they had been successfully trained to draw from more than one field to address a conservation problem. In doing so, they articulated the application of theory to practice. However, what appeared a more arduous task for them was conceptual integration of knowledge from more than one field. Since exogenous interdisciplinarity has led to "an appreciation of conceptual ties" (Klein, 1990, p. 42) in some other areas, we could perhaps predict with some certainty that these fledgling members may manifest more conceptual interdisciplinarity as they become more established members of this disciplinary community.

\section{Works Cited}

Bazerman, C. (1988). Shaping written knowledge: The genre and activity of the experimental article in science. Madison, WI: The University of Wisconsin Press.

Belcher, D. (1995). Writing critically across the curriculum. In D. Belcher \& G. Braine (Eds.), Academic writing in a second language: Essays on research and pedagogy (pp. 135-154). Norwood, NJ: Ablex

Ceccarelli, L. (1995). A rhetoric of interdisciplinary scientific discourse: Textual criticism of Donzhansky's Genetics and the origin of species. Social Epistemology, 9, 91-111.

Dunlap, L. (1992). Advocacy and neutrality: A contradiction in the discourse of urban planners. In A. Herrington \& C. Moran (Eds.), Writing, teaching, and learning in the disciplines (pp. 213-230). New York: Modern Language Association. 
FalerSweany, M. L. (1995). Spanning rhetoric for a holistic science: James Lovelock's geophysiology. Social Epistemology, 9, 165-174.

Fuller, S. (1993). Philosophy, rhetoric, and the end of knowledge: The coming of science and technology studies. Madison, WI: The University of Wisconsin Press.

Hay, P. R., Todd, J. J., \& Russell, J. A. (1986). Teaching the "Human in Environment": A postgraduate approach at the University of Tasmania. Environmental Education and Information, 5, 119-126.

Henry, J. (1994). A narratological analysis of WAC authorship. College English, 56, 810-824.

Herndl, C. G. \& Brown S. C. (Eds.) (1996) Green culture: Environmental rhetoric in contemporary America. Madison, WI: University of Wisconsin Press.

Hill, S. S., Soppelsa, B. F., \& West, G. K. (1982). Teaching ESL students to read and write experimental research papers. TESOL Quarterly, 16, 333347.

Johns, A. M. (1995). Teaching classroom and authentic genres: Initiating students into academic cultures and discourses. In D. Belcher \& G. Braine (Eds.), Academic writing in a second language: Essays on research and pedagogy (pp. 277-292). Norwood, NJ: Ablex.

Journet, D. (1990). Forms of discourse and the sciences of the mind: Luria, Sacks and the role of narrative in neurological case histories. Written Communication, 7, 171-199.

Journet, D. (1993). Interdisciplinary discourse and "boundary rhetoric": The case of S. E. Jelliffe. Written Communication, 10, 510-541.

Journet, D. (1995). Synthesizing disciplinary narratives: George Gaylord Simpson's Tempo and mode in evolution. Social Epistemology, 9, 113150.

Killingsworth, J., \& Palmer, J. S. (1992a). Ecospeak: Rhetoric and environmental politics in America. Carbondale, IL: Southern Illinois University Press.

Killingsworth, J., \& Palmer, J. S. (1992b). How to save the earth: The greening of instrumental discourse. Written Communication, 9, 385403.

Killingsworth, J., \& Steffens, D. (1989). Effectiveness in the environmental impact statement. Written Communication, 6, 155-180.

Klein, J. (1990). Interdisciplinarity: History, theory, and practice. Detroit: Wayne State University Press.

Miller, C. (1980). Environmental impact statements and rhetorical genres: An application of rhetorical theory to technical communication. Ph.D. Thesis. Rennselaer Polytechnic Institute.

Miller, C. (1984). Genre as social action. Quarterly Journal of Speech, 70, 151-167. 
Montgomery, S. (1996). Scientific voice. New York: Guilford Press.

Noss, R. F. (1993). Whither conservation biology? Conservation Biology, 7, 215-217.

Petrie, H. G. (1992). Interdisciplinary education: Are we faced with insurmountable opportunities? In G. Grant (Ed.), Review of Research in Education, 18 (pp. 299-333). American Educational Research Association.

Prior, P. (1992). Contextualizing writing and response in graduate seminars: A sociohistoric perspective on academic literacies. Unpublished $\mathrm{Ph}$. D. Dissertation. University of Minnesota.

Root, T. L. (1993). Effects of global climate change on North American birds and their communities. In P. M. Kareira, J. G. Kingslover \& R. B. Huey (Eds.), Biotic interactions and global change (pp. 280-292). Sunderland, MA: Sinauer Associates Inc.

Root, T. L. \& Schneider, S. H. (1993). Can large-scale climatic models be linked with multiscale ecological studies? Conservation Biology 7, 256-270.

Samraj, B. (1995). The nature of academic writing in an interdisciplinary field. Unpublished $\mathrm{PhD}$ disseratation. The University of Michigan.

Slovic, S. (1996). Epistemology and rhetoric in American nature writing: Embedded rhetoric and discrete rhetoric. In C. G. Herndl \& S. C. Brown (Eds.), Green culture: Environmental rhetoric in contemporary America (pp. 82-110). Madison, WI: University of Wisconsin Press.

Soule, M. E. (1985). What is Conservation Biology? BioScience, 35, 727734.

Sullivan, D. L. (1995). Migrating across disciplinary boundaries: The case of the periodicity paper of David Raup and John Sepkoski. Social Epistemology, 9, 151-164.

Swales, J. M. (1990). Genre analysis: English in academic and research settings. Cambridge: Cambridge University Press.

Ulman, H. L. (1996). "Thinking like a mountain": Persona, ethos, and judgment in American nature writing. In C. G. Herndl \& S. C. Brown (Eds.), Green culture: Environmental rhetoric in contemporary America (pp. 46-81). Madison, WI: University of Wisconsin Press.

Wignell, P. (1998). Technicality and abstraction in social science. In J. R. Martin \& R. Veel (Eds.), Reading science (pp. 297-326). London: Routledge.

\section{Notes}

${ }^{1}$ We would like to thank one anonymous reviewer for a number of very useful comments. 